\title{
Giant Congenital Melanocytic Nevus in an Afghan Child
}

\author{
Nahid Raufi ${ }^{1}$ and Arash Nemat ${ }^{1}$ \\ ${ }^{1}$ Kabul Medical University
}

October 31, 2021

\begin{abstract}
We report an 8-year-old Afghan female with giant congenital melanocytic nevus (GCMN) which covered the entire back. The GCMN extended to anterolateral parts of the trunk surrounded by multiple satellite melanocytic nevi.
\end{abstract}

\section{Introduction}

Congenital melanocytic nevi $(\mathrm{CMN})$ are cutaneous lesions characterized histologically by benign proliferations of melanocytes ${ }^{1}$. GCMN in compare to CMN are deeper and extend more profound with irregular in margin, dark coloured and covered with coarse hair ${ }^{2}$. Neurocutaneous melanoma is associated with GCMN which indicated bad prognosis of the disease ${ }^{3}$.

Here we report a case of GCMN in a child in Afghanistan to highlight this pathology to the attention of clinicians. Although a recent report was published about an Afghan child with GCMN in Iran ${ }^{4}$, to the best of our knowledge, GCMN has not been reported from inside Afghanistan.

\section{Case Report}

An 8 year-old Afghan child who was born in Ghazni province of Afghanistan, presented to our outpatient dermatology clinic with an extensive pigmented plaque over her body since birth. Her parents are first cousins with family history of spotted melanocytic nevi in her brother. Due to lack healthcare access she did not present until 8 years of age. When the lesions became symptomatic with dryness and pruritus, her parents sought evaluation and treatment. Her parents noted that she had asked many times about the dark spots and was distressed about them.

On examination, she had a dark brown melanocytic nevus with hairy rough surface that covered the entire back, reaching both anterolateral aspects of her trunk. (Shown in Fig. 1)

Multiple satellite melanocytic nevi were also observed on her face, chest, and abdomen. No clinically atypical lesions were noted in the GCMN or satellite nevus. She did not have any neurological deficits with no radiological evidence for neural involvement. The patient had a depressed affect.

She was prescribed emollient and sunscreen. We referred the patient to the psychology department for evaluation and treatment of her depression. Unfortunately the patient did not receive further investigations and treatment at dermatology department of our hospital.

\section{Discussion}

GCMN is a large hyperpigmented lesions commonly present since birth, these patches are trend to malignant transformation in the first 3 to 5 years of patients' life ${ }^{5,6}$. The GCMN is occurred 1 in 20000 to 1 in 50000 births ${ }^{2}$. Due to its tendency to the malignant transformation, increased risk of neurological abnormalities and cosmetic reasons the timely treatment is essential ${ }^{7,8}$. Laser therapy, Skin grafting, the curettage of the 
nevus tissue in newborns, and the cultured epidermal autograft (CEA) are reported for GCMN treatment ${ }^{7}$. The treatment options mostly recommended in the early ages of the patients ${ }^{9}$.

To our knowledge, there is no published data from the Ministry of Public Health (MoPH) of Afghanistan on the prevalence of the CMN in the country. Less knowledge of the people, limited access of rural residents to health settings, and poverty let the patients to delay in their visit to the central clinics. Meanwhile for those who present their illness it's difficult to accept the clinicians' prescriptions particularly who need to cost and surgery ${ }^{10}$. In the reality the primary indication for such giant nevus, with high-risk phenotypical features, large size and axial location and multiple satellite lesions of the trunk, the serial excision of the lesion was recommended but unfortunately the patient was lost to follow up.

\section{Statement of Ethics}

This study protocol was reviewed and approved Kabul University of Medical Sciences research committee, approval number was 21-0714 KUMS-RC.

\section{Patient Consent}

The authors certify that they have obtained all appropriate patient consent forms. The parents of the child provided informed consent for publication of the case details and images. Institutional approval is not required for this case study.

\section{Conflict of Interest Statement}

The authors have no conflicts of interest to declare.

\section{Funding Sources}

The case was reported without any financial support.

\section{Author Contributions}

The authors fulfil the ICMJE Criteria for Authorship and contributed equally.

\section{Data Availability Statement}

The all data and images are available inside the paper.

\section{References}

1 Hashmi, H. M., Shamim, N., Kumar, V. \& Idrees, S. Giant Congenital Melanocytic Nevi in a Pakistani Newborn. Cureus 13(2021).

2 Ganjoo, S., Gupta, N., Sawhney, M. P. S. \& Kaur, P. Bathing trunk nevus with multiple satellite lesions and neuroid proliferations. Journal of Pakistan Association of Dermatologists 31 , 304-307 (2021).

3 Puthiyakam, J., Riyaz, N., Arakkal, F. R. \& Arakkal, R. Neurocutaneous melanoma in association with giant congenital melanocytic nevi in a child (Touraine syndrome)-A dermatoradiological correlation. Journal of Skin and Sexually Transmitted Diseases 3 , 84-86 (2021).

4 Mohaghegh, F., Asilian, A., Nabavinejad, S. \& Rezaei, M. Giant cerebriform melanocytic nevus of the scalp: A case report. Clinical Case Reports (2021).

5 Arneja, J. S. \& Gosain, A. K. Giant Congenital Melanocytic Nevi.Plastic and Reconstructive Surgery 120 (2007).

6 Kugar, M. et al. Malignant Melanoma Arising From a Giant Congenital Melanocytic Nevus in a 3-Year Old: Review of Diagnosis and Management. Journal of Craniofacial Surgery 32 (2021).

7 Takaya, K. et al. Clinical Analysis of Cultured Epidermal Autograft (JACE) Transplantation for Giant Congenital Melanocytic Nevus.Plast Reconstr Surg Glob Open 9 , e3380-e3380, doi:10.1097/GOX.0000000000003380 (2021). 
8 Abdulmajid, L. et al. An update on Congenital Melanocytic Nevus Syndrome: a case report and literature review. Journal of Cutaneous Pathology (2021).

9 Carmen Ceballos-Rodríguez, M., Redondo, P., Tomás-Velázquez, A., Cieza-Díaz, D. \& Carlos López-Gutiérrez, J. Surgical outcomes and psychosocial impact of giant congenital melanocytic nevus surgery: A single-center case series of 136 patients. Journal of Pediatric Surgery , doi:https://doi.org/10.1016/j.jpedsurg.2020.12.023 (2021).

10 McMichael, J. \& Lawley, L. P. Propranolol for infantile hemangiomas in developing countries. Dermatology online journal 23(2017).

\section{Figure Legends}

Fig. 1. (A ) Confluent dark brown plagues of the back (B ) Extension to the anterolateral aspects of the trunk with hypertrichosis, with multiple satellite melanocytic nevi on the chest and abdomen. 